\title{
Hydrodynamic Delivery of Chitosan-Folate-DNA Nanoparticles in Rats with Adjuvant-Induced Arthritis
}

\author{
Qin Shi, ${ }^{1}$ Huijie Wang, ${ }^{1}$ Covi Tran, ${ }^{1}$ Xingping Qiu, ${ }^{2}$ Françoise M. Winnik, ${ }^{2}$ Xiaoling Zhang, ${ }^{3,4}$ \\ Kerong Dai, ${ }^{3,4}$ Mohamed Benderdour, ${ }^{1}$ and Julio C. Fernandes ${ }^{1}$ \\ ${ }^{1}$ Orthopaedics Research Laboratory, Research Centre, Sacré-Coeur Hospital, University of Montreal, \\ 5400 West Gouin Boulevard, Montreal, \\ QC, Canada H4J 1 C5 \\ ${ }^{2}$ Faculty of Pharmacy and Department of Chemistry, University of Montreal, CP 6128 Succursale Centre Ville, Montreal, \\ QC, Canada H3C $3 J 7$ \\ ${ }^{3}$ The key laboratory of Stem Cell Biology, Institute of Health Sciences, Shanghai Jiao Tong University School of \\ Medicine (SJTUSM), Shanghai 200025, China \\ ${ }^{4}$ Shanghai Institutes for Biological Sciences (SIBS), Chinese Academy of Sciences (CAS), 225 South Chongqing Road, \\ Shanghai 200025, China
}

Correspondence should be addressed to Qin Shi, qin.shi@umontreal.ca

Received 14 September 2010; Accepted 29 November 2010

Academic Editor: Oreste Gualillo

Copyright ( 2011 Qin Shi et al. This is an open access article distributed under the Creative Commons Attribution License, which permits unrestricted use, distribution, and reproduction in any medium, provided the original work is properly cited.

$50 \mathrm{kDa}$ chitosan was conjugated with folate, a specific tissue-targeting ligand. Nanoparticles such as chitosan-DNA and folatechitosan-DNA were prepared by coacervation process. The hydrodynamic intravenous injection of nanoparticles was performed in the right posterior paw in normal and arthritic rats. Our results demonstrated that the fluorescence intensity of DsRed detected was 5 to 12 times more in the right soleus muscle and in the right gastro muscle than other tissue sections. $\beta$-galactosidase gene expression with X-gal substrate and folate-chitosan-plasmid nanoparticles showed best coloration in the soleus muscle. Treated arthritic animals also showed a significant decrease in paw swelling and IL- $1 \beta$ and $\mathrm{PGE}_{2}$ concentration in serum compared to untreated rats. This study demonstrated that a nonviral gene therapeutic approach using hydrodynamic delivery could help transfect more efficiently folate-chitosan-DNA nanoparticles in vitro/in vivo and could decrease inflammation in arthritic rats.

\section{Introduction}

Human diseases can be treated by the transfer of therapeutic genes (transgene) into specific cells or tissues of patients to correct or supplement defective, causative genes. The success of gene therapy depends on the ability to deliver therapeutic DNA or siRNA or other genetic materials to target site. Viral vectors (e.g., adenovirus) are very effective in term of transfection efficiency, but they have limitations in vivo, particularly by their safety concern and nontissue-specific transfection. Nonviral gene transfer systems are limited by their lower gene transfer efficiency, low tissue specificity, and transient gene expression $[1,2]$. Cationic polymers, such as chitosan $(\mathrm{Ch})$, are promising candidates for DNA transport in nonviral delivery systems $[3,4]$. The enabling characteristics of Ch-DNA nanoparticles include biocompatibility, multiple ligand affinity, and a capacity of taking up large DNA fragments, while remaining small in size [5]. Genes can be delivered systemically (intramuscularly, intravenously, subcutaneously, or, in animals, intraperitoneally). Otherwise, hydrodynamic-based gene delivery offers a convenient, efficient, and powerful means for high-level gene expression in animals [6-8]. The highly effective delivery of naked plasmid into body or tissue with hydrodynamic limb vein injection technique has already been demonstrated for the treatment of muscular dystrophy [9]. However, the mechanism of hydrodynamic injection aiding the delivery of chitosan-based nanoparticles into targeted tissue and the use of this approach in other disease, treatment such as arthritis is unclear. 
Rheumatoid arthritis (RA) is a chronic, systemic autoimmune disorder that causes the immune system to attack the joints, where it causes inflammation (arthritis) and destruction. Other problems may also develop, including inflammation of blood vessels (vasculitis), the development of bumps (called rheumatoid nodules) in various parts of the body, lung disease and blood disorders. The recent report demonstrated that the muscle strength loss is strongly associated with the presence and duration of an inflammatory state like rheumatoid arthritis [10]. Interleukin1 (IL-1), a key proinflammatory factor which consists of 2 subtypes, including IL- $\alpha$ and IL- $1 \beta$, is produced by monocytes macrophages. IL- $1 \alpha$ remains mostly intracellular (or expressed on the cell surface), while IL-1 $\beta$ is secreted into the extracellular space [11]. Prostaglandin $\mathrm{E}_{2}\left(\mathrm{PGE}_{2}\right)$ is considered to be of great importance in pathology of RA [12]. IL- $1 \beta$ has been implicated in the pathological mechanisms of synovial tissue proliferation and joint destruction in RA [2, 11, 13]. Its physiologically competitive agent, IL1 receptor antagonist (IL-1Ra), has been proven to act as a powerful inhibitor [14]. Several therapeutic studies on the use of recombinant IL-1Ra, as an external source agent, have demonstrated its positive effect in controlling inflammation and symptoms of RA in animal models and in clinical practice $[15,16]$. Folate receptors (FR) are overexpressed on many human cancer cell surfaces, and the nonepithelial isoform $\operatorname{FR}(\beta)$ is expressed on activated synovial macrophages present in large numbers in RA [17]. Folate-mediated transfection has been shown to facilitate DNA and nanoparticle internalization into cells through membrane receptors for higher transfection yields both in vitro and in vivo [18].

In this study, $50 \mathrm{kDa}$ chitosan was conjugated with folic acid (Fa), a specific tissue-targeting ligand. Nanoparticles such as chitosan-DNA (Ch-DNA) and Chitosan-folate-DNA (Ch-Fa-DNA) were prepared by coacervation process. The hydrodynamic limb intravenous injection of nanoparticles was performed in the right posterior paw in rats. The first objective was to determine the distribution of these nanoparticles in normal rats following hydrodynamic injection. The second objective of the study was to examine the in vivo anti-inflammaory effects of nanoparticles of plasmid IL-1Ra DNA complexed to the $\mathrm{Ch}$ or $\mathrm{Ch}-\mathrm{Fa}$ in an adjuvant-induced arthritis (AIA) rat model.

\section{Materials and Methods}

All animal experiments were approved by the Animal Ethics Committee of the Hôpital du Sacré-Coeur de Montréal.

2.1. Synthesis of Chitosan-Folate Conjugate. Chitosan (Ch) (Wako-10, degree of deacetylation (DD) $85 \%, \mathrm{M}_{\mathrm{w}}=57 \mathrm{kDa}$ ) was purchased from Wako Chemicals USA (Richmond, VA). Folic acid $(\mathrm{Fa})$ was purchased from Sigma-Aldrich Chemical (St-Louis, MO, USA). $\alpha$-amino, $\omega$-carboxyl poly(ethylene glycol) $\left(\mathrm{NH}_{2}\right.$-PEG-COOH, $\mathrm{M}_{\mathrm{w}}=3,400$ Dalton) was purchased from Shearwater Inc. (Dallas, TX, USA). Ch was further deacetylated by treating with concentrated $\mathrm{NaOH}$ solution (50\%) to obtain $\sim 96 \%$ deacetylation degree according to the reported procedure [19]. To prepare the Ch$\mathrm{Fa}$ conjugate, folic acid was first attached to $\mathrm{NH}_{2}-\mathrm{PEG}$ $\mathrm{COOH}$ via the well-known carbodiimide chemistry to obtain folate-PEG-COOH. Then, the folate-PEG-COOH was again activated by $N, N^{\prime}$-Dicyclohexylcarbodiimide (DCC) and $\mathrm{N}$-hydroxysuccinimide (NHS) to convert to the reactive intermediate folate-PEG-CO-NHS and subsequently grafted onto chitosan to achieve the folate-PEG-chitosan conjugate [20]. The level of folate-PEG incorporation was determined to be $1.1 \mathrm{~mol} \%$ with respect to the glucosamine unit of chitosan by UV-Vis spectroscopy using folic acid as standard (the extinction coefficient $\left(\lambda_{363 \mathrm{~nm}}\right)$ of folic acid is determined to be $6165 \mathrm{M}^{-1} \mathrm{~cm}^{-1}$ in $\mathrm{pH} 7.4$ phosphate buffer $\left.(0.1 \mathrm{M})\right)$.

2.2. IL-Ra Plasmid Construction. We used human interleukin 1 receptor antagonist as a curing agent, with its secretive variant (sIL-1Ra, 177aa, NM_173842 X52015, GenBank). We subcloned this gene by PCR with oligos carrying HindIII and XbaI sites (IL-1RaH3 CCAAGCTTGAATGGAAATCTGCAGAGGCC, IL-1RaH4 GCTCTAGACTGGGCA GTACTACTCGTCCTCC), using genomic DNA of macrophage differentiated from monocyte cell line THP1 stimulated with PMA as PCR DNA template. Then, PCR product was inserted in the mammalian expression vector pCDNA3 (Invitrogen, Burlington, ON, Canada) that drives overexpression of gene with CMV promoter. To reduce the size of pDNA, the region of PvuII-PvuII was deleted. Final plasmid $(3.8 \mathrm{~kb})$ including targeted gene was verified by DNA sequencing.

2.3. Amplification and Purification of Plasmid DNA. The other plasmids used in this study were VR1412 and pDsRed. The plasmid DNA VR1412, encoding the $\beta$-galactosidase $(\beta$ gal) reporter gene with a CMV promoter and a backbone size of $8,100 \mathrm{~kb}$, was obtained from VICAL Inc. (San Diego, CA, USA). The plasmid pDsRed encoding the red fluorescent protein was obtained from Clonetech (Mountain View, CA, USA).

The plasmid DNAs were multiplied with Escherichia Coli bacteria then isolated and purified using the QIAGEN Plasmid Mega Kit according to the manufacturer's instruction (Qiagen, Mississauga, ON, Canada). The purified plasmid was resuspended in water. The purity was confirmed with $0.8 \%$ agarose gel electrophoresis. The DNA concentration was measured by UV absorption at $260 \mathrm{~nm}$ with a UVvisible 1601 spectrophotometer from Shimadzu (Columbia, MD, USA). All bacteria were all grown in GIBCO Invitrogen Corporation Lennox L Broth Base (Invitrogen Canada Inc., Burlington, ON, Canada).

2.4. Nanoparticle Synthesis. Ch or Ch-Fa was dissolved with low heating (below $45^{\circ} \mathrm{C}$ ) in a $20-\mathrm{mM}$ acetic solution at $\mathrm{pH}$ 5.5. The solution was then adjusted to a final concentration of $0.02 \%$ chitosan in $5 \mathrm{mM}$ acetic acetate and passed through a $0.22-\mu \mathrm{m}$ filter. The plasmid DNA ( $\beta$-gal, DsRed, and IL1 Ra plasmid DNAs) employed for synthesis was a solution of $200 \mu \mathrm{g} \mathrm{DNA} / \mathrm{ml}$ of $4.3 \mathrm{mM}$ sodium sulfate solution. After 
heating to $55^{\circ} \mathrm{C}, 100 \mu \mathrm{l}$ of the DNA solution was added to $100 \mu \mathrm{l}$ of Ch or Ch-Fa solution and vortexed immediately at maximum speed for $1 \mathrm{~min}$ [21]. The nanoparticle solutions produced were deployed for stirring 30 minutes and allowing standing one hour before transfection without further modification. Two kinds of nanoparticles were synthesized: Ch-DNA and Ch-Fa-DNA.

2.5. In Vitro Tranfection Test. KB cells obtained from American Type Culture Collection (ATCC) (Rockville, MD, USA) were used for transfection in the media with or without folic acid supplement. On 24-well plates at a density of 50,000 cells/well in $1 \mathrm{ml}$ of Roswell Park Memorial Institute (RPMI), 1640 medium supplemented with 10\% fetal bovine serum (FBS) and $1 \%$ penicillin-streptomycin (PS). Next day, medium was replaced with fresh RPMI medium 1640 or RPMI medium 1640 without folic acid, without FBS in two type media. After 2 hours, KB cells received $500 \mu \mathrm{l}$ solution of Ch- or Ch-Fa- plasmid IL-1Ra DNA nanoparticles (containing $5 \mu \mathrm{g}$ human IL-1Ra plasmid DNA) in the presence or absence of folic acid supplement. After another two hours, $0.5 \mathrm{ml}$ complete RPMI 1640 medium was added in each well. The plates were kept in a cell cultural incubator with $5 \% \mathrm{CO}_{2}$ at $37^{\circ} \mathrm{C}$. After 24 hours, cell culture media and cells were collected separately for later analysis.

2.6. Hydrodynamic Limb Vein Injection of Nanoparticles. THE hydrodynamic intravenous injection was performed in the right posterior paw, as previously published [22-24]. Nanoparticle solutions were adjusted to physical osmolarity with $18 \%$ sterile $\mathrm{NaCl}$ just before injection. The injection point was located in the great saphenous vein inside the leg, in direction of the knee. A tourniquet was located above the knee forcing the solution back into the smaller leg veins and ending up in muscle tissue cells. The $4 \mathrm{ml}$ transfection solution (containing $200 \mu \mathrm{g}$ DNA) was injected into about $200 \mathrm{~g}$ rats, at a speed of $10 \mathrm{ml} /$ minute, with infusion/withdrawal pump Model 940 (Harvard Apparatus, Millis, MA, USA). The cuff was kept in place for one minute after injection. The eyes were humidified with Liquifilm Tears eyes drops from Allergan (Markham, ON, Canada).

2.7. In Vivo Organ Distribution Study by DsRed Protein Fluorescence Measurements. The female Lewis rats were injected with the hydrodynamic technique via the femoral vein with DesRed plasmids DNA, coding for fluorescent emitting proteins, measurable by detecting the emitted DsRed fluorescence wave, with a maximum excitation at $555 \mathrm{~nm}$ and maximal emission at $583 \mathrm{~nm}$. We used 9 female Lewis rats to administer DsRed plasmid (containing $200 \mu \mathrm{g}$ DNA/rat) alone via the hydrodynamic technique by the femoral vein, towards the direction of the knee. 3 experimental groups formed 3 rats for control, 3 rats for hydrodynamic injection, and 3 rats for normal femoral intravenous injection. The rats were sacrificed 6 to 8 days following the injection; the tissue sections of liver, kidney, gluteus superficialis muscle, and different skeletal muscle groups of two hind limbs (femoris bicep, soleus, and gastro) were removed, cleaned by dipping it in clean phosphate buffered saline (PBS) solution, homogenised with Polytron, and centrifuged at $3000 \mathrm{rpm}$. The samples suspensions were then stored in $-80^{\circ} \mathrm{C}$ until the day the measurements are done. The fluorescence density was detected by Photon Technology International (PTI) spectrofluorometer (Birmingham, NJ, USA). Protein determination was performed by the bicinchoninic acid method [25].

2.8. $\beta$-Gal Staining with X-Gal Substrate in Soleus Muscle. The female Lewis rats were injected with the hydrodynamic technique via the femoral vein with $200 \mu \mathrm{g}$ naked $\beta$-gal plasmids DNA ( 3 rats), Ch- $\beta$-gal plasmids DNA (3 rats) and Ch-Fa- $\beta$-gal plasmids DNA (3 rats), respectively. The rats were sacrificed 3 days following the injection. The sampled tissues (soleus muscles) were to be dissected into an ice cold PBS solution containing $2 \mathrm{mM} \mathrm{MgCl}_{2}$. The fixation process is done by dipping them, for 30 minutes and more, in a solution composed of $0.5 \%$ glutaraldehyde, reputed for its superior staining and endogenous enzyme preservation activity, up to the confounding point of the signal, compared to the other way by using paraformaldehyde. It is important to note that the enzyme coding $\beta$-gal can be overfixed. Afterwards, it is very important to carefully rinse of residual fixatives, which can inhibit the enzyme, by rinsing them many times with PBS. At some point, overnight rinsing can be done. The X-gal stock was diluted into the X-gal Reaction Buffer $(40 \mathrm{mg} / \mathrm{ml}$ in die methyl). The tissues were then incubated in it for $2-4$ hours at $37^{\circ} \mathrm{C}$ before getting rinse in PBS. The viewing step was done under the bright fluorescent white light for optimal visualisation and analysis. One of section of specimens were embedded in paraffin, sectioned, and stained for $\beta$-galactosidase protein expression stained with its corresponding polyclonal antibody (Sigma-Aldrich, St-Louis, MO, USA) after hydrodynamic Ch-Fa- plasmid $\beta$-gal DNA injection. The staining was undertaken with DAKO liquid $\mathrm{DAB}+$ substrate-chromogen system kit (DAKO, Carpinteria, CA, USA) as directed by the manufacturer. Each section was examined under a light microscope (Zeiss Axio-Imager Z1, Carl Zeiss MicroImaging GmbH, Göttingen, Germany) at x20 magnification and photographed with a Zeiss AxioCam camera and Axio-Vision software (version 4.0).

2.9. Adjuvant-Induced Arthritis (AIA) Rat Model. Arthritis was induced in 150 to $200 \mathrm{~g}$ female Lewis rats (Charles Rivers, Montreal, QC, Canada). Briefly, on day 1, rats were anaesthetized with $0.2 \mathrm{ml} 0.5 \%$ ketamine and $0.5 \%$ xylazine hydrochloride, and $0.5 \mathrm{mg}$ of heat-killed $\mathrm{M}$ butyrium suspended in mineral oil ( $5 \mathrm{mgml}$, Difco, MI, USA) was injected intradermically into the right hind foot. Disease develops around 10-45 days after injection and generally subsides after a month. Arthritis usually develops in all treated animals, as evidenced by dramatic swelling in the injected paws and progressive swelling in all uninjected paws.

2.10. Experimental and Control Groups under Study. A control group (naked DNA, $n=5$ ), a Ch-plasmid IL-1Ra DNA nanoparticles group $(n=5)$, and a Ch-Fa-plasmid IL1 Ra DNA nanoparticles group $(n=7)$ were injected on day 


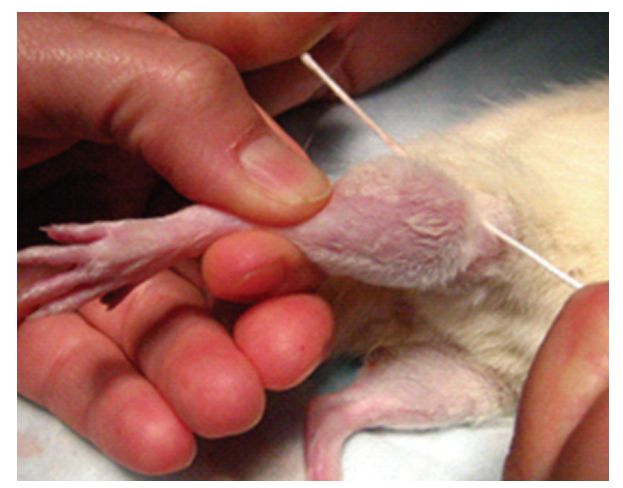

Figure 1: Hydrodynamic injection on hindlimb of rat. The experimental detail of hydrodynamic injection procedure was given in section of Materials and Methods.

18 post AIA induction. A nontreated group (RA) $(n=5)$ was used as an untreated control. All animals were sacrificed on day 35 and clinical results were compared among the groups. The evaluation of reduced adjuvant-induced arthritis was done by evaluating ankle inflammation, decreased articular index scores, ankle circumferences, and sequential calliper measurements of the ankle joints.

2.11. Blood Samples. While under anesthesia, 7-9 $\mathrm{ml}$ of whole rat blood was obtained by heart puncture. Blood was gelled at room temperature then sera were separated by centrifugation at $6000 \mathrm{rpm}$ for 10 minutes. Sera were kept separately at $-80^{\circ} \mathrm{C}$ until assays.

\subsection{Enzyme-Linked Immunosorbent Assay (ELISA) Analysis.} IL-1Ra detection in culture medium and rat sera was carried out with ELISA kit (R\&D Systems, Minneapolis, MN, USA). Blood serum samples were diluted 5-10 times in assay diluents (BioFX, Owings Mills, MD, USA). 100ul of dilution were added in 96 wells plates. No special treatment was necessary for standard series. The rest of the process followed the kit provider's protocol. Rat $\mathrm{PGE}_{2}$ and Il-1 $\beta$ were detected with ELISA kit (R\&D Systems), by diluting blood serum samples 100 times with PBS buffer containing 1\% bovine serum albumin (BSA).

2.13. Statistical Analyses. All values are expressed as means $\pm \mathrm{SD}$ and were subjected to $t$-test and one-way ANOVA analysis. A value of 0.05 was considered significant.

\section{Results}

3.1. Nanoparticle Characterization. All nanoparticle size averaged $108 \mathrm{~nm}$. Zeta potential remained stable for all nanoparticles between +4 and $+6 \mathrm{mV}$.

\subsection{Efficient Hydrodynamic Limb Intravenous Injection. Rats} were injected with the hydrodynamic technique (Infusion/Withdrawal Pump Model 940 from Harvard Apparatus co., inc., Millis, Mass., USA) via the right femoral vein,

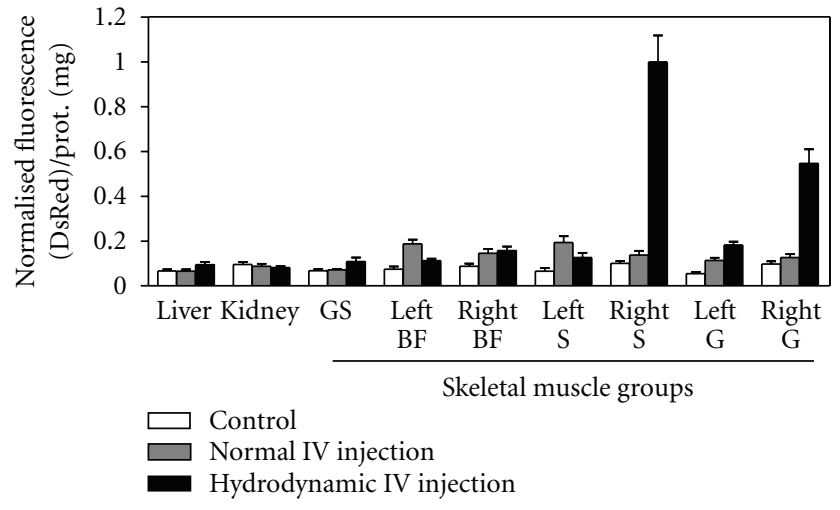

FIGURE 2: In vivo organ distribution study by DsRed protein fluorescence measurements. The normal female Lewis rats were allocated to 3 groups and each consisting of 3 animals. One group, serving as normal controls, received $4 \mathrm{ml}$ of saline injected with the hydrodynamic technique via the femoral vein. Another two Groups received $200 \mu \mathrm{g}$ DsRed plasmids DNA prepared with $4 \mathrm{ml}$ saline injected by normal femoral vein injection and hydrodynamic femoral vein injection, respectively. The rats were sacrificed 6 to 8 days following injection. The tissue sections of liver, kidney, and skeletal muscle were removed, cleaned, and homogenised. The samples suspensions were then stored in $-80^{\circ} \mathrm{C}$ until the day the measurements are done. The DsRed plasmids DNA, coding for fluorescent emitting proteins, were measured by detecting the emitted DsRed fluorescence wave, with a maximum excitation at $555 \mathrm{~nm}$ and maximal emission at $583 \mathrm{~nm}$. The skeletal muscle groups included gluteus superficialis (GS), Femoris Bicep of left and right hindlimbs (Left BF and Right BF), soleus of left and right hindlimbs (Left $S$ and Right $S$ ), and gastro of left and right hindlimbs (Left $G$ and Right $G$ ). Values were shown as mean \pm SEM of normalised DsRed fluorescence/protein $(\mathrm{mg})$ of 3 rats of each group. All values were subjected to t test and one-way ANOVA analysis. A value of 0.05 was considered significant. The highest value of fluorescent spectra (right soleus muscle section) is set as 1 .

toward the direction of the knee (Figure 1). Before performing the injection, a small cut will be done on the skin covering the vein. This lowers the difficulty level when it comes to inserting the needle into the vein. In addition, the leg is bound tightly, above the knee level with a tourniquet to force the solution back into the smaller leg veins and end up in tissue cells. This is very crucial since that is what causes the hydrodynamic pressure, hence the whole point of this type of injection. Once the needle is properly inserted, the injection machine is turned on and its speed is set to three, around 0.3$0.4 \mathrm{ml} / \mathrm{sec}$. After the injection, only the needle is taken out; the elastic binder stays for an extra minute to let the plasmid settle in. For each rat, around $4 \mathrm{ml}$ of the injection solution is needed; as a precaution, $5 \mathrm{ml}$ is set per rat.

3.3. Organ Distribution of Plasmid DNA by Hydrodynamic Limb Intravenous Injection. To determine the tissue uptake of plasmid DNA following hydrodynamic injection, we used 9 female Lewis rats to administer DsRed plasmid alone via the hydrodynamic technique by the femoral vein, towards the direction of the knee. 3 experimental groups formed 3 rats for control, 3 rats for hydrodynamic injection, and 3 


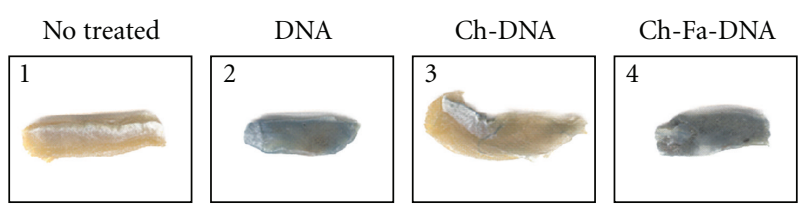

(a)

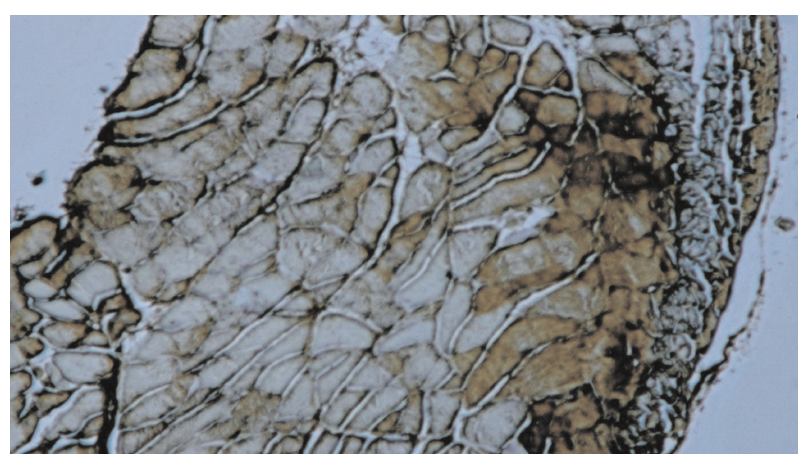

(b)

Figure 3: $\beta$-gal staining with X-Gal substrate in soleus muscle. Four groups (each consisted of 3 rats) of the normal Lewis rats treated with the hydrodynamic femoral intravenous injection of saline or $200 \mu \mathrm{g}$ naked $\beta$-gal plasmid, Ch- $\beta$-gal plasmid DNA, and $\mathrm{Ch}-\mathrm{Fa}$ $\beta$-gal plasmid DNA, respectively. Soleus muscle were isolated and visualized for $\beta$-gal gene expression with $\mathrm{X}$-gal substrate incubation 3 days post injection. (a) Shows the scanned images from the rat soleus muscles. (1) nontreated controls, (2) naked plasmid $\beta$-gal DNA injected, (3) Ch-plasmid $\beta$-gal DNA nanoparticles injected, and (4) the Ch-Fa-plasmid $\beta$-gal DNA nanoparticles injected rats. (b) Shows soleus muscle histological cross-sectional patterns of $\beta$-galactosidase protein expression stained with its corresponding antibody after hydrodynamic $\mathrm{Ch}$-Fa- plasmid $\beta$-gal DNA injection (dark brown staining).

rats for normal femoral intravenous injection. The rats were sacrificed 6 to 8 days following the injection; specific DsRed plasmid expressed red fluorescent protein expression was measured by spectrofluorometer in various tissue extracts (Figure 2). The highest level of red fluorescent protein was observed in the soleus muscle of the right legs of hydrodynamic injected rat group. The different sampled organs also show much lower red fluorescence intensities, like the liver that emits 12 times less. Lastly, by comparing the hydrodynamic injected rats with the control and normal intravenous injected rats, the majority of the red fluorescence values are higher for hydrodynamic injected rats.

In order to study $\mathrm{Ch}$ or $\mathrm{Ch}-\mathrm{Fa}$ as gene delivery vector, three groups (each consisted of 3 rats) of the normal Lewis rats treated with the hydrodynamic intravenous injection of $200 \mu \mathrm{g}$ naked $\beta$-gal plasmid, Ch- $\beta$-gal plasmid DNA, and $\mathrm{Ch}-\mathrm{Fa}-\beta$-gal plasmid DNA, respectively. Soleus muscles were isolated and visualized with $\mathrm{X}$-gal expression 3 days post injection. As shown in Figure 3, the naked DNA can be expressed (Figure 3(a)2). The Ch-Fa-DNA expression (Figure 3(a)4) is darkest in the soleus muscle compared to Ch-DNA expression (Figure 3(a)3). Figure 3(b) showed soleus muscle histological cross-sectional patterns of $\beta$-gal protein expression stained with its corresponding antibody after hydrodynamic injection of nanoparticles of Ch-Faplasmid $\beta$-gal DNA with positive dark-brown staining within the muscle.

3.4. Efficient Delivery of Ch-Fa-IL-1Ra Plasmid DNA In Vitro and In Vivo. In vitro, to test the modification by adding folic acid on chitosan backbone, the nanoparticles made of two types of chitosan were transfected in KB cells with medium without folic acid supplement. Data showed the significantly higher expressive level in 1640 without folic acid medium $(n=3, P<.01)$ comparing to that in normal medium (Figure 4(a)). Evidently folic acid in medium interferes the binding between folate-modified nanoparticle and cells.

In vivo, as shown in this Figure 4(b), the naked plasmid is more rapidly expressed in greater quantity in the serum than the Ch-nano and Ch-Fa-nanoparticles. Despite the longer time taken by Ch-DNA and Ch-Fa-DNA nanoparticles has taken the longer time to express themselves in the serum, but they presented more secreted IL-1Ra proteins at the day of 34 with compared to naked DNA treated rats. However, there were no significant differences in the IL-1Ra protein concentration across the three treatment groups (naked DNA, Ch-DNA, and Ch-Fa-DNA).

\subsection{Efficacy in a Rat Model of Arthritis. Paw swelling.} Decrease in macroscopic inflammation in various degrees in AIA rat's paws after treatment with Ch-plasmid IL1Ra DNA, or Ch-Fa-plasmid IL-1Ra DNA, as shown in Figure 5(a). There is a decrease in the percentage of ankle size in all treated groups compared to the untreated RA positive control group. Only the Ch-Fa-pIL-1Ra group showed significant decrease in ankle size $(P<.05, n=$ $5)$. However, there was no significant difference between all other treatment groups (naked DNA, and Ch-DNA).

Levels of serum proinflammatory factor Il-1 $\beta$ and $P G E_{2}$. To measure the inflammation changes after treatment, cytokine IL-1 $\beta$, a potent immunomodulator which mediates a wide range of immune and inflammatory responses, was detected in the serum of the experimental animal rat model. A gradual increase of cytokine level was seen in the positive control group (RA), while there was an abrupt decrease of IL- $1 \beta$ levels following treatment with Ch-plasmid IL-1Ra DNA and Ch-Fa-plasmid IL-1Ra DNA nanoparticles. This bring to the fore the inflammation reduction, since IL-1Ra competes for the IL- 1 receptor and indirectly decrease IL- $1 \beta$ production therein (Figure 5(b)). As shown in Figure 5(c), a gradual increase of $\mathrm{PGE}_{2}$ levels is seen in the positive control group (RA) whereas there is a decrease of $\mathrm{PGE}_{2}$ levels following treatment with Ch-pIL-1Ra. Lower levels of $\mathrm{PGE}_{2}$ synthesis were observed in all treated groups compared to positive control.

\section{Discussion}

The hydrodynamic limb vein injection involves the rapid injection of a large volume of naked DNA through a peripheral vein a limb that is temporarily isolate from normal blood flow by a tourniquet [6-8]. In this study, 


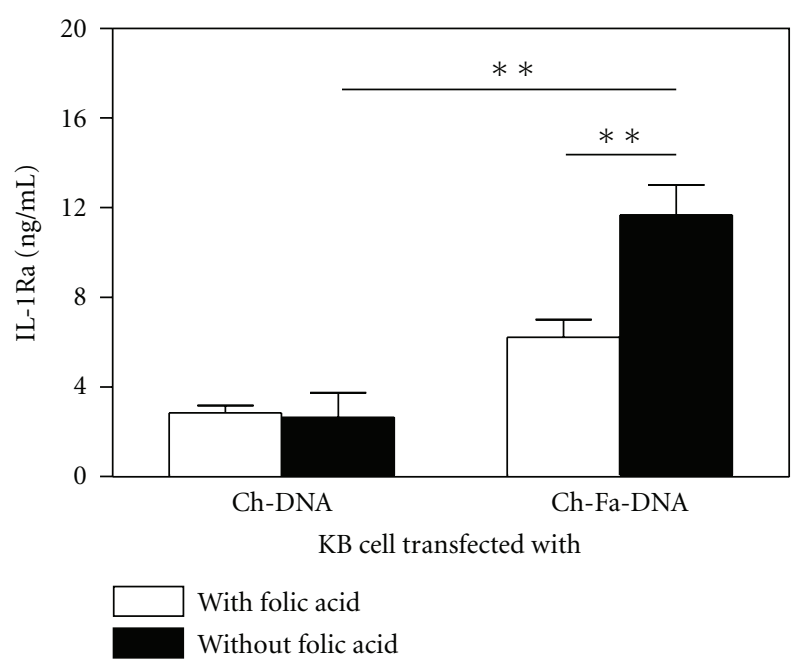

(a)

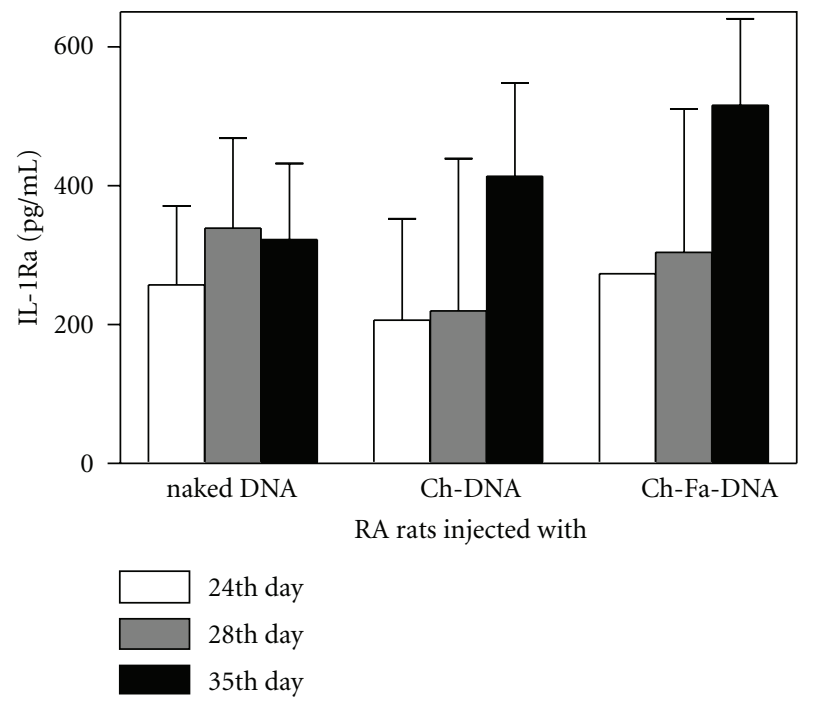

(b)

FIgUre 4: Efficient delivery of Ch-Fa-plasmid IL-1Ra DNA in vitro and in vivo. (a) In vitro, $\mathrm{KB}$ cells received either $5 \mu \mathrm{g}$ of Chplasmid IL-1Ra DNA nanoparticles or Ch-Fa-plasmid IL-1Ra DNA nanoparticles in the presence or absence of folic acid supplement. Values are the mean \pm SEM. Statistical significance was assessed by unpaired Student $t$. (b) Three groups (each consisted of 3 rats) of the normal Lewis rats treated with the hydrodynamic femoral intravenous injection of $200 \mu \mathrm{g}$ naked $\beta$-gal plasmid, Ch- $\beta$-gal plasmid DNA, and Ch-Fa- $\beta$-gal plasmid DNA, respectively. Serum samples were collected at day 24, 28 and 35, respectively. The levels of IL-1Ra protein in the cellular cultured media and serum were analyzed by ELISA. Values were shown as mean \pm SEM of three rats. All values were subjected to $t$ test and one-way ANOVA analysis. A value of 0.05 was considered significant.

we found that the expression of the targeted protein by a Ch-nanoparticle is possible (as reflected by DeRed protein expression and staining by $\beta$-gal in the soleus muscle). Although hydrodynamic injection causes edema at the site of puncture (data not show), injection skills may affect the effect of genetic material delivered [26]. However, in our study, this manipulation was performed by the same person, used to the technique, thereby reducing the effect of the learning curve on the quality of delivery. In addition, the hydrodynamic injection allows the introduction of high volume solution into the muscle tissue. This situation allows for a greater concentration of nanoparticles around cells suggesting a greater potential of capture through endocytosis. The transfection efficiency of nonviral vectors may depend on several factors such as chemical structure of polycations, size and composition of complexes, interaction between cells and complexes and the cell type. The small size complexes have the advantage of entering the cells through endocytosis or pinocytosis and crossing the nuclear pore complex, thereby increasing the transfection rate. Our results demonstrated that our nanoparticles (average $108 \mathrm{~nm}$ ) obtained favored nanosize. Many researchers [27, 28], including us [29], are actually working on the synthesis of smaller size nanoparticles.

The hydrodynamic injection showed 5 to 12 times more red protein fluorescence in the leg muscles in proximity of the injected femoral vein than the other sampled parts (ex: kidney). It even goes up to 20 times more when comparing with the control (ex: left gastro muscle). This is what we hoped to obtain with the hydrodynamic vein tourniquet injection. A normal femoral vein injection done using these same manipulations, the results would not appear much in the legs, but instead it will mostly be in the rat's liver and spread across other body parts. In our case, the tourniquet made so that the solution travels back into smaller veins and end up in muscle cells. In the case where the tourniquet is well installed, directing the process towards good conditions by blocking the escape, the local injection load is administered gently enough to trap and spread the injected solution in and not beyond the tourniquet. If some of the solution gets to escape, it would get filtered, stopped, in the lungs, liver, kidneys, and aorta, and in most cases, travel in the blood circulation system. That is why in rat with normal intravenous injection, we were not able to observe the leg muscles swelling (data not shown). Instead, the whole leg got bigger in size, acting like a balloon being blown up. Probably, the solution was at first trapped in the leg due to the tourniquet applied in the lymphatic and vein drainage system which stop the solution from spreading beyond the knee with high pressure. The pressure does so that the injected solution in the femoral vein stops going forward and is forced back into the smaller veins in the opposite direction.

It has been demonstrated that naked DNA express easily when injected into a muscle mass [30]. According to the results obtained through $\beta$-gal staining with $\mathrm{X}$-gal, we can see that without any treatment, the naked DNA can be expressed. When surrounded by a layer of $50 \mathrm{kDa}$ chitosan as a nonviral approach, only fine blue lines can be seen on part of the targeted soleus leg muscle. This means that the particles entered difficultly into the cells beyond the veins surrounding the leg muscle. The answer to where they went is found by observing the final destination of the body fluid circulation, acting like body "filter", the liver [31-33]. Once upgraded with folic acid ( $\mathrm{Fa})$, the results are totally different. The soleus muscles are suitably darker than the control. Evidently, 


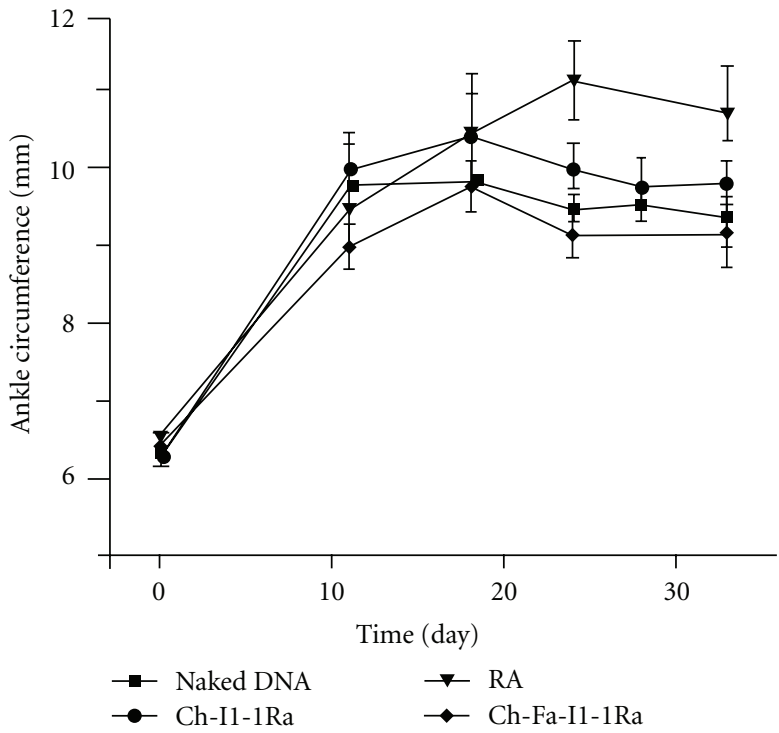

(a)

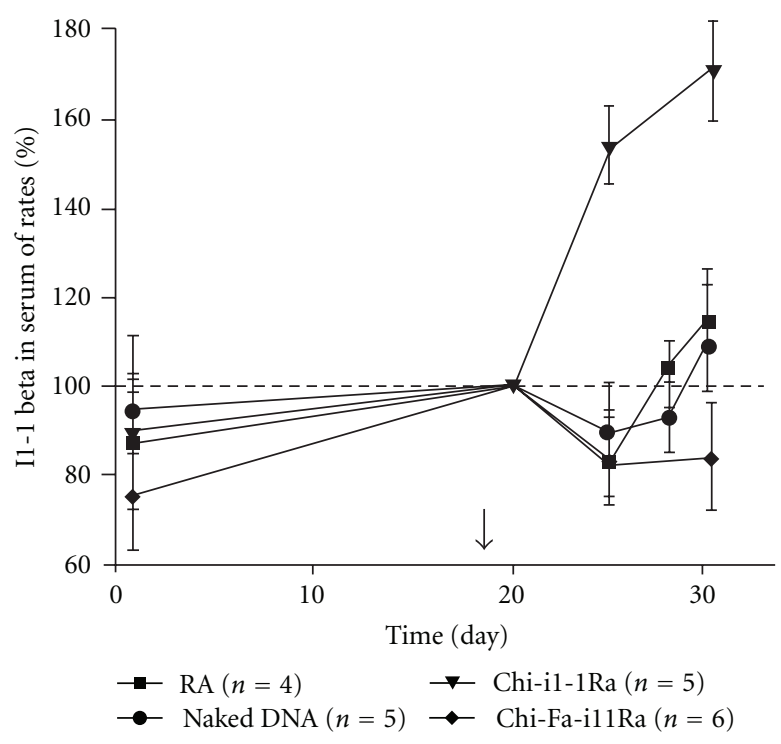

(b)

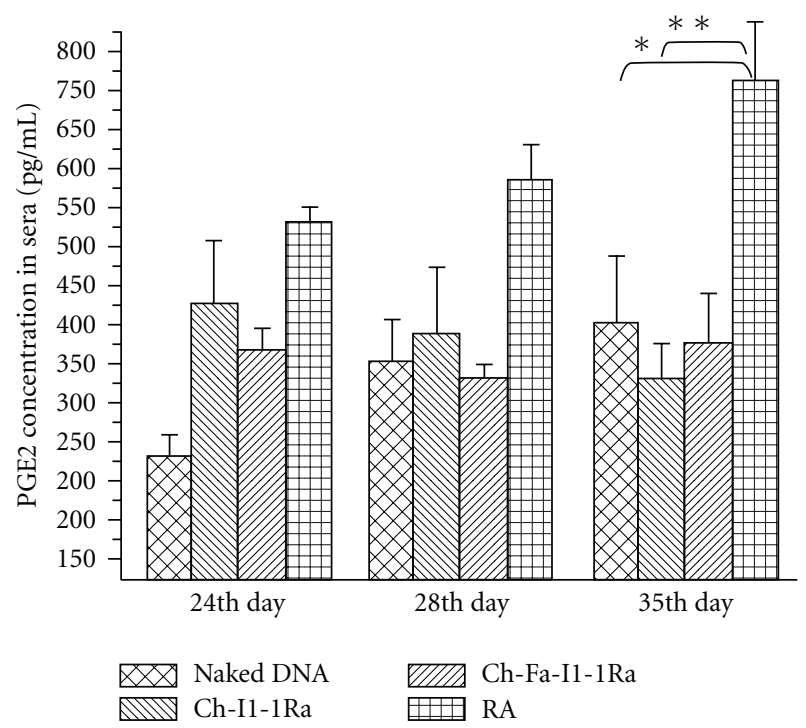

(c)

FIGURE 5: Efficacy in a rat model of arthritis. The rats were inoculated intradermally, into the plantar surface of the right hindpaw, with $0.1 \mathrm{ml}$ of complete Freund's adjuvant (CFA) containing $10 \mathrm{mg} \mathrm{ml}^{-1}$ of heat-killed Mycobacterium tuberculosis suspended in paraffin oil. The rats were allocated to 5 groups. A control group (naked DNA, $n=5$ ), a natural Ch-IL-1Ra plasmid DNA nanoparticles group ( $n=5$ ) and a Ch-Fa-IL-1Ra plasmid DNA nanoparticles group $(n=7)$ were injected on day 18 post AIA induction. A nontreated group (Rheumatoid arthritis, RA) $(n=5)$ was used as an untreated control. All animals were sacrificed on day 35 and clinical results were compared among the groups. (a) Rat's ankle size change before and after treatments. Graph shows changes of absolute ankle size (mm) in AIA rats before and after different treatments at day 18 . Each point represents the mean \pm SD. Note a significant decreasing inflammation with Ch-Fa-Il-1Ra gene therapy $(P \leq .05, n=7)$ comparing to the AIA nontreated control. Differences observed between all treated groups were nonsignificant (NS). (b) IL- $1 \beta$ concentration in rat sera. All treated groups showed significant decrease IL-1Ra in serum $(P \leq .05, n=5)$ compared to nontreated controls. It can be clearly seen that this effect is observed the day after treatment starts. Black arrow indicates the day of starting IL-1Ra gene therapy. (c) $\mathrm{PGE}_{2}$ level in rat sera after Il-1Ra treatment. All values are expressed as means \pm SD and were subjected to $t$ test and one-way ANOVA analysis. A value of 0.05 was considered significant. There is a significant decrease in the levels of PGE $\mathrm{in}_{2}$ all three treatment groups $(* P<.05, * * P<.01)$. 
the intensity is lower in the liver (data not shown). When observing the hydrodynamic injection technique efficiency rate for this experiment, the injections technical skill play a very important role.

We observed that IL-1Ra protein expression was significantly higher $(P<.05)$ when transfection was prepared with chitosan-folate-IL-1Ra plasmid DNA complexes in the culture medium without folic acid supplement (Figure 4(a)). These findings suggested that folic acid molecules supplemented in culture medium would prevent cellular uptake of the complexes by competitive binding to FRs on the cell surface. The in vivo study revealed that the IL-1Ra gene can be transduced in vivo exploiting nonviral chitosan and folate target technology via hydrodynamic limb vein injection. The injected muscle was the predominant site of gene transfer and expression after hydrodynamic limb vein injection. In our study, Il-1Ra protein expression was also detected in rat sera. It is interesting to note that IL-1Ra protein can be detected in the serum of rats as long as 6-17 days following the injections of both chitosan-DNA and chitosan-folate-DNA nanoparticles, possibly offering a lasting, sustain release of IL-1Ra in the serum. Folate conjugation improves the transfection efficiencies of FAchitosan for FR-positive cell gene silencing in conditions without folic acid supplement. Because of FR overexpression in cancer cells and activated macrophages, a variety of therapeutic agents have been linked with folic acid for selective delivery to pathological cells, by avoiding normal tissues. Among these therapies, folate-targeted nonviral gene therapy vectors are considered as promising treatments of cancer and inflammatory diseases [33-35].

The hydrodynamic limb vein injection technology has been shown to be effective for the delivery naked DNA into the skeletal muscle of the limb. This approach has a good profile for the treatment of muscular disease such as muscular dystrophy. In this study, the usefulness of hydrodynamic delivery to decrease inflammation in experimental arthritis treatment was also demonstrated. The first model of RA to be described was adjuvant arthritis, and the arthritis develops 10-45 days after injection and generally subsides after a month [36]. Based on our experiences with adjuvant induced arthritis in rats, disease is allowed to obtain maximum inflammation around 18 days, as evidenced by dramatic swelling in the injected paw measurements of proinflammatory biomarkers such as TNF- $\alpha$ and IL-1 $\beta$. So we inject chitosan/folate-chitosan-DNA nanoparticles at day 18 to treat inflammation caused by adjuvant injection.Our results showed that it is possible to express the IL-1Ra protein with a variety of cationic polymeric-based nanoparticles. Macroscopically, hydrodynamic injection of these nanoparticles in an AIA rat model allow a significant decrease of the inflammation in the rats' ankle compared to untreated rats, proving indirectly the efficacy of the IL- $1 \mathrm{Ra}$ protein treatment. Various inflammation markers (IL-1 $\beta$ and $\mathrm{PGE}_{2}$ ) showed a significant decrease in the muscle and serum after the injection of the IL-1Ra protein demonstrating by direct evidence the efficacy of the administration technique to deliver efficient nanoparticles. Therefore, it is possible to do gene therapy via hydrodynamic limb vein injection with
IL-1Ra to decrease arthritis and have a positive effect on inflammation.

In conclusion, the efficacy of chitosan-folate-based nanovectors was confirmed in vitro in $\mathrm{KB}$ cells and in vivo using hydrodynamic delivery of $\beta$-gal DNA nanocarriers in Lewis rats. This study demonstrated the efficacy of a therapeutic approach (after maximum disease progression) using hydrodynamic delivery of plasmid IL-1Ra DNA and chitosan-DNA-based nanocarriers in controlling the progression of inflammation in an AIA rat model. More research are needed toward the search for smaller and more performing nanoparticles in the treatment of arthritis using gene therapy.

\section{Acknowledgments}

This work was supported by the Grants from National Natural Science Foundation of China (no. 30811120440 and 30911120261), The Ministry of Science and Technology of China (no. S2011GR0323), Shanghai International Collaboration Foundation (no. 08410701800) and Canadian Institutes of Health Research (CCI-92212, CCL-99636, and CCM 104888). J. C. Fernandes and M. Benderdour are research scholars of Fonds de la Recherche en santé du Québec (FRSQ).

\section{References}

[1] R. W. Herzog, O. Cao, and A. Srivastava, "Two decades of clinical gene therapy-success is finally mounting," Discovery Medicine, vol. 9, no. 45, pp. 105-111, 2010.

[2] M. A. Mintzer and E. E. Simanek, "Nonviral vectors for gene delivery," Chemical Reviews, vol. 109, no. 2, pp. 259-302, 2009.

[3] T. Kean and M. Thanou, "Biodegradation, biodistribution and toxicity of chitosan," Advanced Drug Delivery Reviews, vol. 62, no. 1, pp. 3-11, 2010.

[4] H. Tong, Q. Shi, J. C. Fernandes, LI. Liu, K. Dai, and X. Zhang, "Progress and prospects of chitosan and its derivatives as nonviral gene vectors in gene therapy," Current Gene Therapy, vol. 9, no. 6, pp. 495-502, 2009.

[5] S. Techaarpornkul, S. Wongkupasert, P. Opanasopit, A. Apirakaramwong, J. Nunthanid, and U. Ruktanonchai, "Chitosan-mediated siRNA delivery in vitro: effect of polymer molecular weight, concentration and salt forms," AAPS PharmSciTech, vol. 11, no. 1, pp. 64-72, 2010.

[6] G. Zhang, V. Budker, and J. A. Wolff, "High levels of foreign gene expression in hepatocytes after tail vein injections of naked plasmid DNA," Human Gene Therapy, vol. 10, no. 10, pp. 1735-1737, 1999.

[7] F. Liu, Y. K. Song, and D. Liu, "Hydrodynamics-based transfection in animals by systemic administration of plasmid DNA," Gene Therapy, vol. 6, no. 7, pp. 1258-1266, 1999.

[8] K. Kamimura, G. Zhang, and D. Liu, "Image-guided, intravascular hydrodynamic gene delivery to skeletal muscle in pigs," Molecular Therapy, vol. 18, no. 1, pp. 93-100, 2010.

[9] G. Zhang, C. I. Wooddell, J. O. Hegge et al., "Functional efficacy of dystrophin expression from plasmids delivered to mdx mice by hydrodynamic limb vein injection," Human Gene Therapy, vol. 21, no. 2, pp. 221-237, 2010.

[10] K. G. M. Beenakker, C. H. Ling, C. G. M. Meskers et al., "Patterns of muscle strength loss with age in the general 
population and patients with a chronic inflammatory state," Ageing Research Reviews, vol. 9, no. 4, pp. 431-436, 2010.

[11] G. Spohn, I. Keller, M. Beck, P. Grest, G. T. Jennings, and M. F. Bachmann, "Active immunization with IL-1 displayed on virus-like particles protects from autoimmune arthritis," European Journal of Immunology, vol. 38, no. 3, pp. 877-887, 2008.

[12] J. M. McCoy, J. R. Wicks, and L. P. Audoly, "The role of prostaglandin E2 receptors in the pathogenesis of rheumatoid arthritis," Journal of Clinical Investigation, vol. 110, no. 5, pp. 651-658, 2002.

[13] S. B. Mizel, J. M. Dayer, S. M. Krane, and S. E. Mergenhagen, "Stimulation of rheumatoid synovial cell collagenase and prostaglandin production by partially purified lymphocyteactivating factor (interleukin 1)," Proceedings of the National Academy of Sciences of the United States of America, vol. 78, no. 4, pp. 2474-2477, 1981.

[14] Z. Liao, R. S. Grimshaw, and D. L. Rosenstreich, "Identification of a specific interleukin 1 inhibitor in the urine of febrile patients," Journal of Experimental Medicine, vol. 159, no. 1, pp. 126-136, 1984.

[15] M. Stolina, S. Adamu, M. Ominsky et al., "RANKL is a marker and mediator of local and systemic bone loss in two rat models of inflammatory arthritis," Journal of Bone and Mineral Research, vol. 20, no. 10, pp. 1756-1765, 2005.

[16] R. Goldbach-Mansky, "Blocking interleukin-1 in rheumatic diseases: its initial disappointments and recent successes in the treatment of autoinflammatory diseases," Annals of the New York Academy of Sciences, vol. 1182, pp. 111-123, 2009.

[17] M. J. Turk, G. J. Breur, W. R. Widmer et al., "Folate-targeted imaging of activated macrophages in rats with adjuvantinduced arthritis," Arthritis and Rheumatism, vol. 46, no. 7, pp. 1947-1955, 2002.

[18] S. Sabharanjak and S. Mayor, "Folate receptor endocytosis and trafficking," Advanced Drug Delivery Reviews, vol. 56, no. 8, pp. 1099-1109, 2004.

[19] S. Mima, M. Miya, R. Iwamoto, and S. Yoshikawa, "Highly deacetylated chitosan and its properties," Journal of Applied Polymer Science, vol. 28, no. 6, pp. 1909-1917, 1983.

[20] K. C. Cho, S. H. Kim, J. H. Jeong, and T. G. Park, "Folate receptor-mediated gene delivery using folate-poly(ethylene glycol)-poly(L-lysine) conjugate," Macromolecular Bioscience, vol. 5, no. 6, pp. 512-519, 2005.

[21] T. Kiang, J. Wen, H. W. Lim, and K. W. Leong, "The effect of the degree of chitosan deacetylation on the efficiency of gene transfection," Biomaterials, vol. 25, no. 22, pp. 52935301, 2004.

[22] H. Toumi, J. Hegge, V. Subbotin et al., "Rapid intravascular injection into limb skeletal muscle: a damage assessment study," Molecular Therapy, vol. 13, no. 1, pp. 229-236, 2006.

[23] J. E. Hagstrom, J. Hegge, G. Zhang et al., "A facile nonviral method for delivering genes and siRNAs to skeletal muscle of mammalian limbs," Molecular Therapy, vol. 10, no. 2, pp. 386398, 2004

[24] G. Danialou, A. S. Comtois, S. Matecki et al., "Optimization of regional intraarterial naked DNA-mediated transgene delivery to skeletal muscles in a large animal model," Molecular Therapy, vol. 11, no. 2, pp. 257-266, 2005.

[25] P. K. Smith, R. I. Krohn, and G. T. Hermanson, "Measurement of protein using bicinchoninic acid," Analytical Biochemistry, vol. 150, no. 1, pp. 76-85, 1985.

[26] H. Toumi, J. Hegge, V. Subbotin et al., "Rapid intravascular injection into limb skeletal muscle: a damage assessment study," Molecular Therapy, vol. 13, no. 1, pp. 229-236, 2006.
[27] H. Q. Mao, K. Roy, V. L. Troung-Le et al., "Chitosan-DNA nanoparticles as gene carriers: synthesis, characterization and transfection efficiency," Journal of Controlled Release, vol. 70, no. 3, pp. 399-421, 2001.

[28] M. Lavertu, S. Méthot, N. Tran-Khanh, and M. D. Buschmann, "High efficiency gene transfer using chitosan/DNA nanoparticles with specific combinations of molecular weight and degree of deacetylation," Biomaterials, vol. 27, no. 27, pp. 4815-4824, 2006.

[29] S. Mansouri, Y. Cuie, F. Winnik et al., "Characterization of folate-chitosan-DNA nanoparticles for gene therapy," Biomaterials, vol. 27, no. 9, pp. 2060-2065, 2006.

[30] U. Stein, W. Walther, A. Stege, A. Kaszubiak, I. Fichtner, and H. Lage, "Complete in vivo reversal of the multidrug resistance phenotype by jet-injection of anti-MDR1 short hairpin RNAencoding plasmid DNA," Molecular Therapy, vol. 16, no. 1, pp. 178-186, 2008.

[31] S. Inoue, Y. Hakamata, M. Kaneko, and E. Kobayashi, "Gene therapy for organ grafts using rapid injection of naked DNA: application to the rat liver," Transplantation, vol. 77, no. 7, pp. 997-1003, 2004.

[32] G. Zhang, X. Gao, Y. K. Song et al., "Hydroporation as the mechanism of hydrodynamic delivery," Gene Therapy, vol. 11, no. 8, pp. 675-682, 2004.

[33] J. Sudimack and R. J. Lee, "Targeted drug delivery via the folate receptor," Advanced Drug Delivery Reviews, vol. 41, no. 2, pp. 147-162, 2000.

[34] P. S. Low and A. C. Antony, "Folate receptor-targeted drugs for cancer and inflammatory diseases," Advanced Drug Delivery Reviews, vol. 56, no. 8, pp. 1055-1058, 2004.

[35] D. Lee, R. Lockey, and S. Mohapatra, "Folate receptormediated cancer cell specific gene delivery using folic acidconjugated oligochitosans," Journal of Nanoscience and Nanotechnology, vol. 6, no. 9-10, pp. 2860-2866, 2006.

[36] R. O. Williams, "Rodent models of arthritis: relevance for human disease," Clinical and Experimental Immunology, vol. 114, no. 3, pp. 330-332, 1998. 


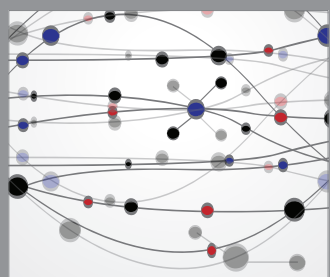

The Scientific World Journal
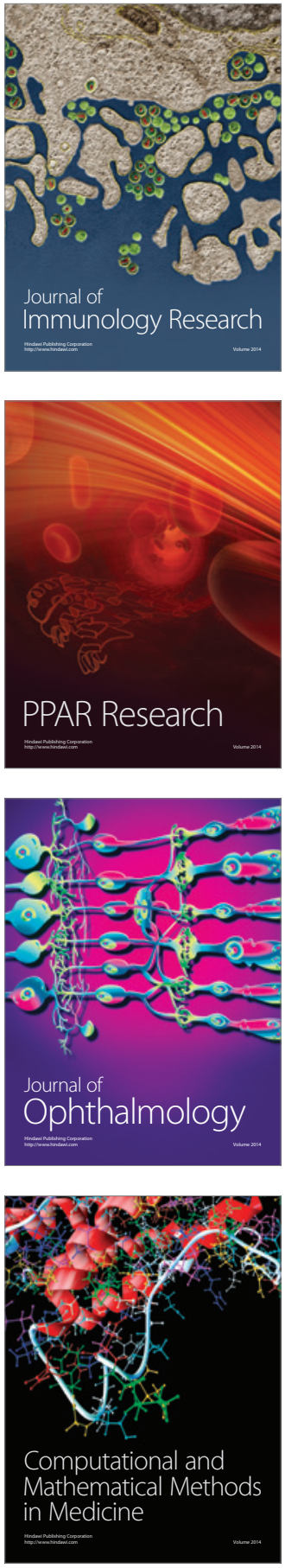

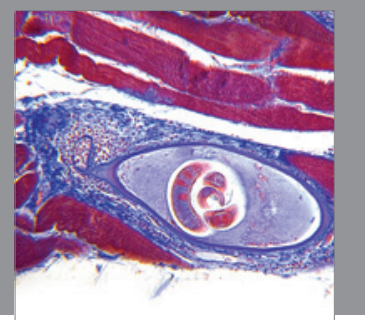

Gastroenterology

Research and Practice
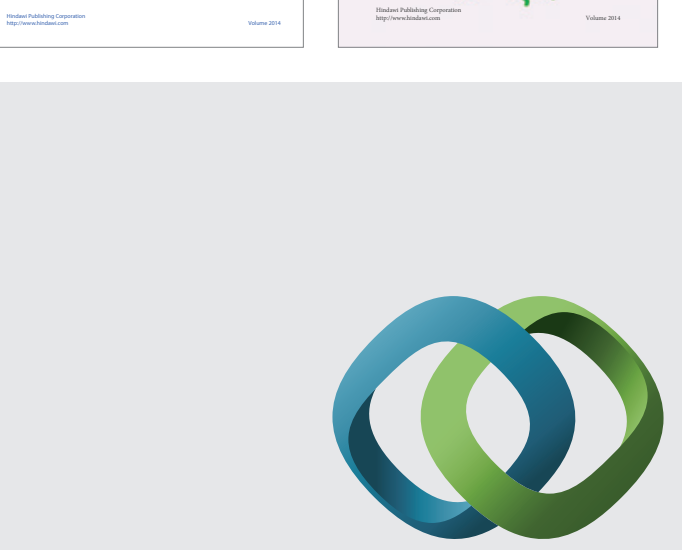

\section{Hindawi}

Submit your manuscripts at

http://www.hindawi.com
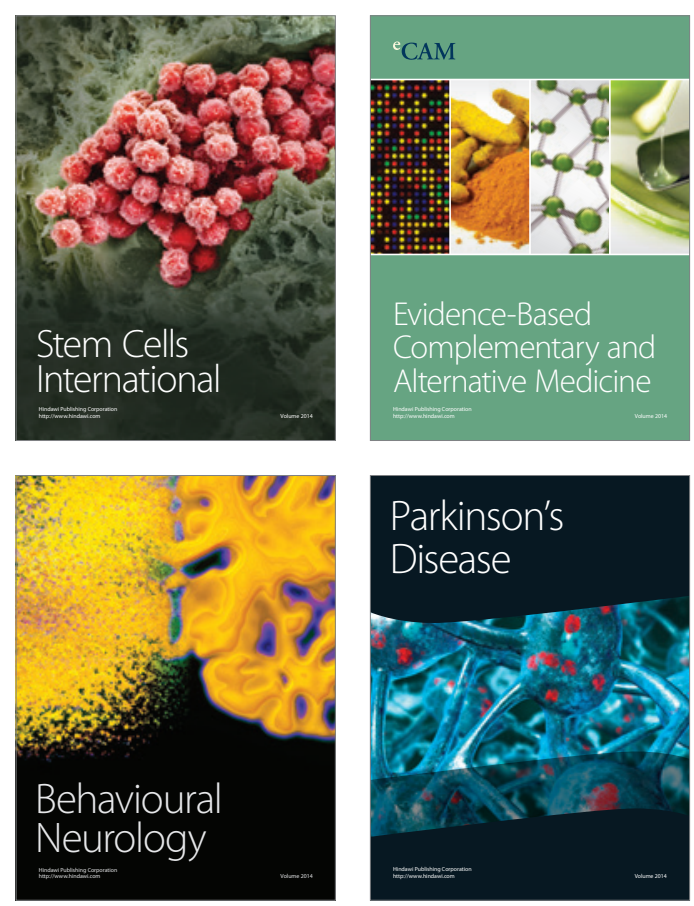

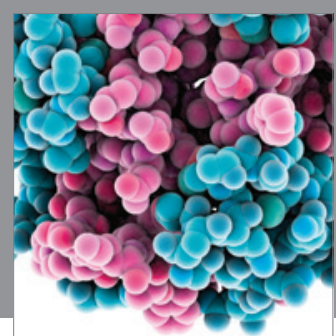

Journal of
Diabetes Research

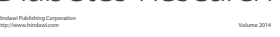

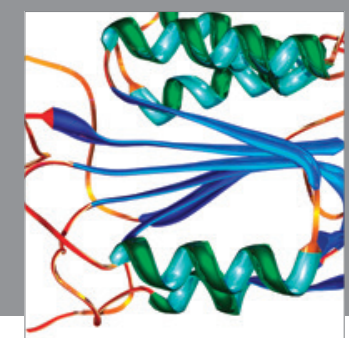

Disease Markers
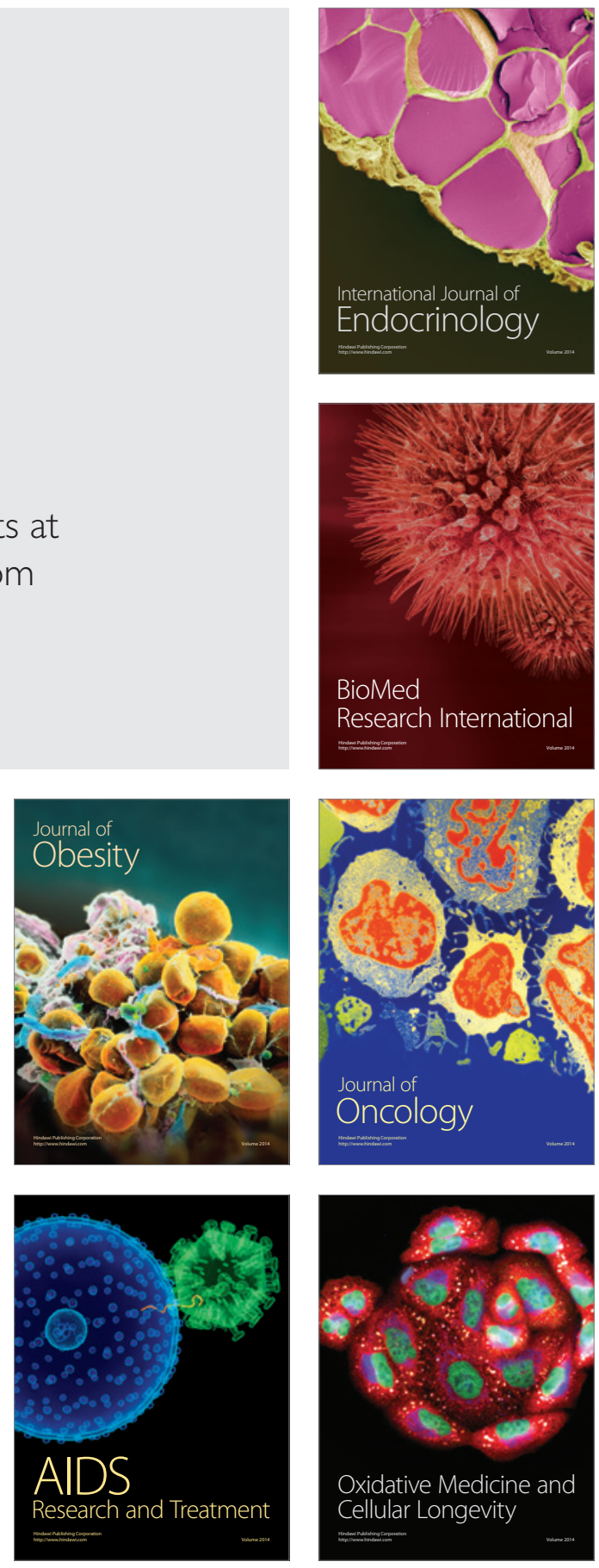